\title{
Development of new hard hat dimensions using user-centered design approach among oil palm harvesters
}

\begin{abstract}
Background: Hard hats are among the personal protective equipment (PPE) used in many industries to reduce the impact of any falling object on the skull and also to prevent head and brain injuries. However, the practice of wearing a safety helmet during working hours is still low. This is due to the physical discomfort perceived by safety helmet users. Objective: Given the unpopularity of the current hard hat, the general perception of workers concerning its use and its measurements are the determining factors in the development of a new hard hat. Method: A cross-sectional study was conducted in which 132 male oil palm harvesters between 19 and 60 years of age were selected from among the employees of the same oil palm harvesting company. A set of questionnaires was developed to collect their sociodemographic information as well as their perceptions of comfort and the prevalence of head injury. In addition, a set of measuring instruments, including Martin's anthropometry set, was used for head measurement and data collection in respect of the current hard hat. In this research, six respondents were randomly selected to attend an interview session for qualitative assessment. Results: Based on the questionnaires, the unpopularity in the use of the hard hat was largely influenced by factors related to poor design, in general, and, specifically, poor ventilation (64\%), load (67\%), and physical discomfort (42\%). The measurements of the anthropometric parameters and the dimensions of the hard hat also showed a significant mismatch. Conclusion: The unpopularity of the current hard hat among oil palm harvesters stemmed from the discomfort from wearing, which showed that the development of a new hard hat could lead to better usage and the greater likelihood of wearing a hard hat throughout the working day.
\end{abstract}

Keyword: Helmet; Harvester; Safety; Comfort 